\title{
Dynamics of the diffusive Nicholson's blowflies equation with distributed delay
}

\author{
S. A. Gourley \\ Department of Mathematics and Statistics, University of Surrey, \\ Guildford, Surrey GU2 5XH, UK (s.gourley@mcs.surrey.ac.uk)
}

\section{S. Ruan}

Department of Mathematics and Statistics, Dalhousie University, Halifax, Nova Scotia, Canada B3H 3J5 (ruan@mscs.dal.ca)

(MS received 26 May 1999; accepted 13 August 1999)

\begin{abstract}
In this paper we study the diffusive Nicholson's blowflies equation. Generalizing previous works, we model the generation delay by using an integral convolution over all past times and results are obtained for general delay kernels as far as possible. The linearized stability of the non-zero uniform steady state is studied in detail, mainly by using the principle of the argument. Global stability both of this state and of the zero state are studied by using energy methods and by employing a comparison principle for delay equations. Finally, we consider what bifurcations are possible from the non-zero uniform state in the case when it is unstable.
\end{abstract}

\section{Introduction}

Over a period of two years, Nicholson [14] carefully recorded the population of the Australian sheep-blowfly (Lucia cuprina) and observed a regular basic periodic fluctuation of $c a .40$ days. Although the delayed logistic equation (see [9])

$$
\frac{\mathrm{d} u}{\mathrm{~d} t}=r u(t)\left(1-\frac{u(t-\tau)}{K}\right)
$$

could be used to explain the oscillatory phenomenon (see [13]), with $K$ representing the food level available and $\tau$ denoting the approximate time for a larva to mature into an adult, the discrepancy is that the Hutchinson's equation gives a delay of $c a .9$ days while the actual delay is $c a .11$ days. To overcome this discrepancy, Gurney et al. [8] proposed the following delay model

$$
\frac{\mathrm{d} u}{\mathrm{~d} t}=-\delta u(t)+p u(t-\tau) \exp [-a u(t-\tau)]
$$

where $p$ is the maximum per capita daily egg production rate, $1 / a$ is the size at which the blowfly population reproduces at its maximum rate, $\delta$ is the per capita daily adult death rate and $\tau$ is the generation time. Since this equation explains Nicholson's data of blowflies more accurately (see [15]), it is now referred to as the Nicholson's blowflies equation and has been studied by many researchers 
(see $[11,17,18]$ and the references cited therein). Notice that after rescaling

$$
u^{*}=a u, \quad t^{*}=\tau t, \quad \tau^{*}=\delta \tau, \quad \beta=p / \delta
$$

and dropping the asterisks, the Nicholson's blowflies equation becomes

$$
\frac{\mathrm{d} u}{\mathrm{~d} t}=-\tau u(t)+\beta \tau u(t-1) \exp [-u(t-1)] .
$$

Recently, the diffusive Nicholson's blowflies equation

$$
\frac{\partial u}{\partial t}=-\tau u(\boldsymbol{x}, t)+\beta \tau u(\boldsymbol{x}, t-1) \exp [-u(\boldsymbol{x}, t-1)]+d \nabla^{2} u
$$

with Dirichlet boundary conditions has been proposed and investigated by So and Yang [19]. They studied the global attractivity of the positive steady state of the equation. Some numerical and Hopf bifurcation analysis of this model has been carried out by So et al. [20] (see also [22] for an analysis of the equation with Neumann boundary conditions).

In this paper we shall study various local and global aspects of the following generalization of (1.1)

$$
\begin{aligned}
\frac{\partial u}{\partial t}=- & \tau u(\boldsymbol{x}, t) \\
& +\beta \tau\left(\int_{-\infty}^{t} f(t-s) u(\boldsymbol{x}, s) \mathrm{d} s\right) \exp \left(-\int_{-\infty}^{t} f(t-s) u(\boldsymbol{x}, s) \mathrm{d} s\right)+d \nabla^{2} u
\end{aligned}
$$

for $(\boldsymbol{x}, t) \in \Omega \times[0, \infty)$, where $\Omega$ is either all of $\mathbb{R}^{n}$ or some finite domain, and where the kernel $f(t)$ satisfies $f(t) \geqslant 0$ and the conditions

$$
\int_{0}^{\infty} f(t) \mathrm{d} t=1 \text { and } \int_{0}^{\infty} t f(t) \mathrm{d} t=1 .
$$

Equation (1.2) is a scalar diffusive equation with distributed (infinite) delay. Such equations have been extensively used to model single species growth with both diffusive and hereditary effects and exhibit very rich and interesting dynamics (see, for example, $[1,3,6,16])$. For the fundamental theories of reaction diffusion equations with delay, we refer to the monograph of $\mathrm{Wu}[21]$.

Equation (1.1) is a special case of (1.2) because the kernel $f(t)$ in (1.2) can be chosen to be $\delta(t-1)$. The first of the conditions (1.3) ensures that the presence of delays does not affect the equilibrium (steady) states. The quantity $\tau$ measures the delay in the system in the sense that an alternative way to formulate the problem is to take $\tau=1 \mathrm{in}(1.2)$, drop the second assumption in (1.3) and then define

$$
\tau=\int_{0}^{\infty} t f(t) \mathrm{d} t
$$

the mean of the delay kernel $f(t)$ (this is the standard way to define the delay in distributed delay models). If one does this and then makes appropriate nondimensionalizations, the result is equation (1.2), with the second assumption in (1.3). This is the sense in which $\tau$ in (1.2) measures the delay. The point is that 
we want $\tau$ to appear explicitly in the model equation, not implicitly through some constraint.

The time delay in the model of the present paper represents generation time. There are numerous other possible reasons for incorporating time delays into models of populations, for example, resource regeneration times and gestation periods (see [5] for a general discussion of the reasons for introducing time delays in population models). Indeed, one can further argue that the incorporation of time delays into a diffusion model should give rise to non-local spatial effects (see [3]). This can be done straightforwardly only if the domain is infinite (in which case the time delayed term will also involve a convolution in the spatial variable). However, in this paper one of our main interests is the question of global convergence and our techniques are only suitable for studying such problems on a finite domain. Thus non-local spatial effects are not included.

The paper is organized as follows. In $\S 2$ we confirm that solutions of our equation which start positive, stay positive. This is important for ecologism realism and positivity of solutions is also needed in certain parts of our mathematical analysis. In $\S 3$ we study the uniform states $u \equiv 0$ and $u \equiv \ln \beta$ of (1.2) in terms of both their local (linearized) stability and their global stability. Global stability is analysed using energy methods and comparison methods, while for the linearized analysis the roots of the dispersion relations are studied by using the principle of the argument. In $\S 4$ we consider what bifurcations might occur from the $\ln \beta$ state when it is unstable, and finally we summarize our conclusions in $\S 5$.

\section{Positivity of solutions}

In models of population dynamics it is important to ensure, for biological realism, that solutions of the model equations remain positive for all time. In this subsection we prove the following theorem.

THEOREM 2.1. If the spatial domain $\Omega$ is finite, with homogeneous Neumann boundary conditions $\nabla u \cdot \boldsymbol{n}=0$ on the smooth boundary $\partial \Omega$, then the solution of (1.2) corresponding to the initial data $u(\boldsymbol{x}, t)=\phi(\boldsymbol{x}, t)$ for $t \leqslant 0, \boldsymbol{x} \in \bar{\Omega}$ will satisfy $u(\boldsymbol{x}, t)>0$ for all $t>0$ and $\boldsymbol{x} \in \bar{\Omega}$, provided that $\phi \geqslant 0$ and $\phi \neq \equiv$.

Proof. Without loss of generality we can assume $u(\boldsymbol{x}, 0)=\phi(\boldsymbol{x}, 0) \not \equiv 0$. Otherwise there must exist an earlier time at which $\phi \not \equiv 0$ and that earlier time can play the role of $t=0$ in the argument below. In this case, the integral terms in (1.2) will play the role of forcing functions from this earlier time until time $t=0$, but our argument still holds.

Suppose the theorem is false, then there is a first time $t^{*}$ and corresponding $\boldsymbol{x}^{*} \in \bar{\Omega}$ such that $u\left(\boldsymbol{x}^{*}, t^{*}\right)=0$. Then the integral terms in (1.2) are non-negative for $t \leqslant t^{*}$ and so

$$
\frac{\partial u}{\partial t}-d \nabla^{2} u+\tau u(\boldsymbol{x}, t) \geqslant 0 \quad \text { for }(\boldsymbol{x}, t) \in \Omega \times\left(0, t^{*}\right] .
$$

The strong maximum principle (see [2, theorem 4.9]) then says that either $u>0$ in $\Omega \times\left(0, t^{*}\right]$ or $u \equiv 0$ in $\Omega \times\left(0, t^{* *}\right]$ for some $t^{* *} \leqslant t^{*}$. The first of these possibilities will contradict $u\left(\boldsymbol{x}^{*}, t^{*}\right)=0$ unless $\boldsymbol{x}^{*} \in \partial \Omega$, a possibility still to be covered, while the second possibility would imply $u(\boldsymbol{x}, 0) \equiv 0$ which contradicts $u(\boldsymbol{x}, 0) \not \equiv 0$. 
If $u\left(\boldsymbol{x}^{*}, t^{*}\right)=0$ with $\boldsymbol{x}^{*} \in \partial \Omega$, then the boundary point lemma says either that $\nabla u \cdot \boldsymbol{n}<0$ at $\left(\boldsymbol{x}^{*}, t^{*}\right)$ (contradicting the boundary conditions) or that $u \equiv 0$ in $\Omega \times\left(0, t^{* *}\right]$ for some $t^{* *} \leqslant t^{*}$ which, as above, contradicts the fact that the initial data is not identically zero. This completes the proof of the theorem.

\section{Stability of the uniform states}

Equation (1.2) has two spatially uniform steady states, these are the zero state and the state $u^{*} \equiv \ln \beta$. Of course, the latter is only ecologically relevant if $\beta>1$ and its stability will be studied under this assumption. In this section we present detailed stability results for each of these two states, starting with the zero state.

\subsection{Global stability of the zero state}

In this subsection we shall discuss the global stability (in the $L^{2}$ norm) of the zero steady state of (1.2) if the domain is bounded and the boundary conditions are homogeneous Neumann.

Theorem 3.1. If $\beta<1$ and the spatial domain $\Omega$ is finite, with homogeneous Neumann boundary conditions $\nabla u \cdot \boldsymbol{n}=0$ on the smooth boundary $\partial \Omega$, then the zero steady state of (1.2) is globally asymptotically stable.

Proof. Letting $u$ be a solution of (1.2), we define

$$
H(t)=\int_{\Omega}(u(\boldsymbol{x}, t))^{2} \mathrm{~d} \boldsymbol{x} .
$$

We differentiate $H(t)$ along solutions of (1.2). Certainly,

$$
\int_{\Omega} u \nabla^{2} u \mathrm{~d} \boldsymbol{x}=\int_{\partial \Omega} u \nabla u \cdot \boldsymbol{n} \mathrm{d} S-\int_{\Omega}|\nabla u|^{2} \mathrm{~d} \boldsymbol{x},
$$

which is clearly negative, since $\nabla u \cdot \boldsymbol{n}=0$ on $\partial \Omega$. Also, since $u$ has been shown to be positive,

$$
\exp \left[-\int_{-\infty}^{t} f(t-s) u(\boldsymbol{x}, s) \mathrm{d} s\right] \leqslant 1 .
$$

Thus it is easily seen that

$$
\frac{1}{2} \frac{\mathrm{d} H}{\mathrm{~d} t} \leqslant-\tau H(t)+\beta \tau \int_{-\infty}^{t} f(t-s) \int_{\Omega} u(\boldsymbol{x}, t) u(\boldsymbol{x}, s) \mathrm{d} \boldsymbol{x} \mathrm{d} s .
$$

Next we use the basic inequality $x y \leqslant \frac{1}{2} \alpha^{2} x^{2}+\left(1 / 2 \alpha^{2}\right) y^{2}$, where $\alpha$ can be chosen to our advantage. The second term in the above is bounded by

$\beta \tau \int_{-\infty}^{t} f(t-s)\left[\int_{\Omega} \frac{1}{2} \alpha^{2}(u(\boldsymbol{x}, t))^{2} \mathrm{~d} \boldsymbol{x}\right] \mathrm{d} s+\beta \tau \int_{-\infty}^{t} f(t-s)\left[\int_{\Omega} \frac{1}{2 \alpha^{2}}(u(\boldsymbol{x}, s))^{2} \mathrm{~d} \boldsymbol{x}\right] \mathrm{d} s$,

which equals, using

$$
\int_{0}^{\infty} f(t) \mathrm{d} t=1
$$


on the first term,

$$
\frac{1}{2} \beta \tau \alpha^{2} H(t)+\frac{\beta \tau}{2 \alpha^{2}} \int_{-\infty}^{t} f(t-s) H(s) \mathrm{d} s .
$$

Thus

$$
\frac{1}{2} \frac{\mathrm{d} H}{\mathrm{~d} t} \leqslant\left(-\tau+\frac{1}{2} \beta \tau \alpha^{2}\right) H(t)+\frac{\beta \tau}{2 \alpha^{2}} \int_{-\infty}^{t} f(t-s) H(s) \mathrm{d} s
$$

or, on choosing $\alpha=1 / \sqrt{\beta}$,

$$
\frac{\mathrm{d} H}{\mathrm{~d} t} \leqslant-\tau H(t)+\beta^{2} \tau \int_{-\infty}^{t} f(t-s) H(s) \mathrm{d} s .
$$

Solutions of this differential inequality are bounded by solutions of the corresponding differential equality, to which the theorems of [12] and [4] apply. The conclusion is that $H(t) \rightarrow 0$ as $t \rightarrow \infty$ if all the roots $\sigma$ of the equation $\sigma+\tau-\beta^{2} \tau \bar{f}(\sigma)=0$ are strictly in the left half of the complex plane, where $\bar{f}$ denotes the Laplace transform of $f$,

$$
\bar{f}(\sigma)=\int_{0}^{\infty} f(t) \mathrm{e}^{-\sigma t} \mathrm{~d} t .
$$

We show that $\beta<1$ ensures that the roots of this equation are where we want them. Suppose that $\beta<1$ but that there is a root $\sigma$ with $\operatorname{Re} \sigma \geqslant 0$. For such a root,

$$
\begin{aligned}
|\bar{f}(\sigma)| & \leqslant \int_{0}^{\infty} f(t)\left|\mathrm{e}^{-\sigma t}\right| \mathrm{d} t \\
& =\int_{0}^{\infty} f(t)\left|\mathrm{e}^{-(\operatorname{Re} \sigma) t}\right| \mathrm{d} t \\
& \leqslant \int_{0}^{\infty} f(t) \mathrm{d} t \\
& =1
\end{aligned}
$$

Since the root satisfies $\sigma+\tau-\beta^{2} \tau \bar{f}(\sigma)=0$, we have

$$
|\sigma+\tau|=\left|\beta^{2} \tau \bar{f}(\sigma)\right| \leqslant \beta^{2} \tau,
$$

so that it lies in the circle centred at $-\tau$ in the complex plane and having radius $\beta^{2} \tau$. But $\beta<1$ implies that this circle is contained entirely in the left half plane, contradicting the assumption that $\operatorname{Re} \sigma \geqslant 0$.

Thus we have shown that if $\beta<1$, then the zero solution of our equation is globally asymptotically stable in the $L^{2}$ norm sense. What we are doing essentially is bounding the $L^{2}$ norm of $u$, namely $H(t)$, in terms of the solutions of a linear differential inequality, so that we end up using linear ideas in obtaining a nonlinear result.

It can easily be shown that if $\beta>1$, then the zero steady state is linearly unstable. Thus our stability condition cannot be improved. 


\subsection{Linearized stability of the non-zero steady state}

The result in the previous subsection indicates that (1.2) undergoes the first bifurcation at $\beta=1$ : when $\beta<1$, the zero steady state is stable; when $\beta>1$, it becomes unstable and a non-zero steady state $u^{*}=\ln \beta$ exists. Throughout the remainder of this paper we shall assume that $\beta>1$ so that this steady state is positive.

If we linearize (1.2) about $u^{*}=\ln \beta$, by setting $u=\ln \beta+\tilde{u}$ and neglecting nonlinear terms in $\tilde{u}$, we obtain

$$
\frac{\partial \tilde{u}}{\partial t}=-\tau \tilde{u}+\tau(1-\ln \beta) \int_{-\infty}^{t} f(t-s) \tilde{u}(\boldsymbol{x}, s) \mathrm{d} s+d \nabla^{2} \tilde{u} .
$$

Trial solutions of the form

$$
\tilde{u}=\exp (\sigma t+\mathrm{i} \boldsymbol{k} \cdot \boldsymbol{x})
$$

exist whenever

$$
\sigma+\tau-\tau(1-\ln \beta) \bar{f}(\sigma)+d k^{2}=0,
$$

where $k^{2}=\boldsymbol{k} \cdot \boldsymbol{k}$ and $\bar{f}$ denotes the Laplace transform of $f$. Of course, if the domain is finite, then $\exp (\mathrm{i} \boldsymbol{k} \cdot \boldsymbol{x})$ is not necessarily the appropriate trial solution, it depends on the shape of the domain. However, the eigenvalue equation will always have the form given by $(3.2), k^{2}$ being an eigenvalue of $-\nabla^{2}$. There will be a countably infinite sequence of eigenvalues if the domain is finite, and any positive value is allowable if it is infinite.

We first prove the following result which gives a local stability condition on the steady state $u^{*}=\ln \beta$ that is independent of the delay $\tau$.

TheOREM 3.2. If $1<\beta \leqslant \mathrm{e}^{2}$, then the steady state $u^{*}=\ln \beta$ of (1.2) is linearly stable for any delay kernel.

Proof. We need to show that all the roots $\sigma$ of (3.2) are in the left half of the complex plane for any $k^{2} \geqslant 0$. Let's assume for contradiction that there is a root with $\operatorname{Re} \sigma \geqslant 0$ for some $k^{2} \geqslant 0$. For this root, $|\bar{f}(\sigma)| \leqslant 1$, so that

$$
\begin{aligned}
\left|\sigma+\tau+d k^{2}\right| & =|\tau(1-\ln \beta) \bar{f}(\sigma)| \\
& \leqslant \tau|1-\ln \beta|
\end{aligned}
$$

and the root lies in the circle centred at $-\tau-d k^{2}$ and having radius $\tau|1-\ln \beta|$. The desired contradiction will certainly be achieved if this circle is entirely in the left half complex plane and, noting that the worst case scenario is $k=0$, this will be the case if $\tau|1-\ln \beta|<\tau$, which gives $1<\beta<\mathrm{e}^{2}$. If $\beta=\mathrm{e}^{2}$, then the abovementioned circle will have radius $\tau$ and so, if $k=0$, it will just include the origin. But it is easily verified that $\sigma=0$ cannot be a root of the eigenvalue equation. This completes the proof.

Next we shall analyse the eigenvalue equation (3.2) in a more general way, and then give some further sufficient stability conditions which follow from this. The eigenvalue equation is $F(\sigma)=0$, where

$$
F(\sigma)=\sigma+\tau-\tau(1-\ln \beta) \bar{f}(\sigma)+d k^{2} .
$$


It follows from a general result in complex variable theory that the number of roots of $F(\sigma)=0$ in the right half of the complex plane will be given by

$$
\lim _{R \rightarrow \infty} \frac{1}{2 \pi \mathrm{i}} \int_{\gamma(R)} \frac{F^{\prime}(\sigma)}{F(\sigma)} \mathrm{d} \sigma,
$$

where $\gamma(R)$ is taken as the closed semicircular contour centred at the origin and contained in $\operatorname{Re} \sigma \geqslant 0$. We let $\gamma_{\mathrm{c}}(R)$ be the curved part

$$
\gamma_{\mathrm{c}}(R)=\left\{\operatorname{Re}^{\mathrm{i} \theta}, \theta \in[-\pi / 2, \pi / 2]\right\}
$$

and $\gamma_{\mathrm{s}}(R)$ be the straight part

$$
\gamma_{\mathrm{s}}(R)=\{\mathrm{i} y, y \in[R,-R]\},
$$

so the contour is traversed in the anticlockwise sense. We shall first show that

$$
\lim _{R \rightarrow \infty} \frac{1}{2 \pi \mathrm{i}} \int_{\gamma_{\mathrm{c}}(R)} \frac{F^{\prime}(\sigma)}{F(\sigma)} \mathrm{d} \sigma=\frac{1}{2} .
$$

To prove this, first note that

$$
\frac{1}{2 \pi \mathrm{i}} \int_{\gamma_{\mathrm{c}}(R)} \frac{\mathrm{d} \sigma}{\sigma}=\frac{1}{2} .
$$

Hence

$$
\begin{aligned}
& \left|\frac{1}{2 \pi \mathrm{i}} \int_{\gamma_{\mathrm{c}}(R)} \frac{F^{\prime}(\sigma)}{F(\sigma)} \mathrm{d} \sigma-\frac{1}{2}\right| \\
& =\frac{1}{2 \pi}\left|\int_{\gamma_{\mathrm{c}}(R)}\left(\frac{F^{\prime}(\sigma)}{F(\sigma)}-\frac{1}{\sigma}\right) \mathrm{d} \sigma\right| \\
& =\frac{1}{2 \pi}\left|\int_{-\pi / 2}^{\pi / 2}\left(\frac{-R \mathrm{e}^{\mathrm{i} \theta} \tau(1-\ln \beta) \bar{f}^{\prime}\left(R \mathrm{e}^{\mathrm{i} \theta}\right)-\tau+\tau(1-\ln \beta) \bar{f}\left(R \mathrm{e}^{\mathrm{i} \theta}\right)-d k^{2}}{R \mathrm{e}^{\mathrm{i} \theta}\left(R \mathrm{e}^{\mathrm{i} \theta}+\tau-\tau(1-\ln \beta) \bar{f}\left(R \mathrm{e}^{\mathrm{i} \theta}\right)+d k^{2}\right)}\right) \mathrm{i} R \mathrm{e}^{\mathrm{i} \theta} \mathrm{d} \theta\right| \\
& \leqslant \frac{1}{2 \pi} \int_{-\pi / 2}^{\pi / 2}\left(\frac{R \tau|1-\ln \beta|\left|\bar{f}^{\prime}\left(R \mathrm{e}^{\mathrm{i} \theta}\right)\right|+\tau+\tau|1-\ln \beta|+d k^{2}}{R-\tau-\tau|1-\ln \beta|-d k^{2}}\right) \mathrm{d} \theta \\
& \leqslant \text { const. } \int_{-\pi / 2}^{\pi / 2}\left|\bar{f}^{\prime}\left(R \mathrm{e}^{\mathrm{i} \theta}\right)\right| \mathrm{d} \theta+O\left(\frac{1}{R}\right) \rightarrow 0 \quad \text { as } R \rightarrow \infty \quad(\text { see below }) .
\end{aligned}
$$

In one of these estimates we have used the fact that $\left|\bar{f}\left(R \mathrm{e}^{\mathrm{i} \theta}\right)\right| \leqslant 1$. The term $\left|\bar{f}^{\prime}\left(R \mathrm{e}^{\mathrm{i} \theta}\right)\right| \leqslant 1$ has to be estimated in a more sensitive way, as follows:

$$
\begin{aligned}
\int_{-\pi / 2}^{\pi / 2}\left|\bar{f}^{\prime}\left(R \mathrm{e}^{\mathrm{i} \theta}\right)\right| \mathrm{d} \theta & =\int_{-\pi / 2}^{\pi / 2}\left|\int_{0}^{\infty}-t f(t) \exp \left(-t R \mathrm{e}^{\mathrm{i} \theta}\right) \mathrm{d} t\right| \mathrm{d} \theta \\
& \leqslant \int_{-\pi / 2}^{\pi / 2} \int_{0}^{\infty} t f(t) \mathrm{e}^{-t R \cos \theta} \mathrm{d} t \mathrm{~d} \theta \\
& =2 \int_{0}^{\infty} t f(t)\left(\int_{0}^{\pi / 2} \mathrm{e}^{-t R \sin \phi} \mathrm{d} \phi\right) \mathrm{d} t \\
& \leqslant 2 \int_{0}^{\infty} t f(t)\left(\int_{0}^{\pi / 2} \mathrm{e}^{-2 t R \phi / \pi} \mathrm{d} \phi\right) \mathrm{d} t
\end{aligned}
$$




$$
\begin{aligned}
& =\frac{\pi}{R} \int_{0}^{\infty} f(t)\left(1-\mathrm{e}^{-t R}\right) \mathrm{d} t \\
& \leqslant \frac{\pi}{R} \rightarrow 0 \quad \text { as } R \rightarrow \infty .
\end{aligned}
$$

In one of these estimates we have used the inequality $\sin \phi \geqslant 2 \phi / \pi$, which is valid for $0 \leqslant \phi \leqslant \pi / 2$.

We now have that

$$
\begin{aligned}
\lim _{R \rightarrow \infty} \frac{1}{2 \pi \mathrm{i}} \int_{\gamma(R)} \frac{F^{\prime}(\sigma)}{F(\sigma)} \mathrm{d} \sigma & =\frac{1}{2}+\lim _{R \rightarrow \infty} \frac{1}{2 \pi \mathrm{i}} \int_{R}^{-R} \frac{F^{\prime}(\mathrm{i} y)}{F(\mathrm{i} y)} \mathrm{i} \mathrm{d} y \\
& =\frac{1}{2}+\lim _{R \rightarrow \infty} \frac{1}{2 \pi \mathrm{i}}(\ln F(-\mathrm{i} R)-\ln F(\mathrm{i} R)) \\
& =\frac{1}{2}-\frac{1}{\pi} \lim _{R \rightarrow \infty} \arg F(\mathrm{i} R) .
\end{aligned}
$$

Here we have used the fact that $F(\mathrm{i} R)$ and $F(-\mathrm{i} R)$ are complex conjugates of each other (as can easily be shown) so their moduli are equal but their arguments differ in sign.

We've now shown that the number of roots of $F(\sigma)=0$ in the right half complex plane is given by the last line in the above calculation. Some further facts will enable us to pin down the various possibilities. First note that

$$
F(0)=\tau-\tau(1-\ln \beta) \int_{0}^{\infty} f(t) \mathrm{d} t+d k^{2}=\tau \ln \beta+d k^{2}>0 .
$$

Also, $F(\mathrm{i} R)=\mathrm{i} R+\tau-\tau(1-\ln \beta) \bar{f}(\mathrm{i} R)+d k^{2}$, so that, since $|\bar{f}(\mathrm{i} R)| \leqslant 1,|\operatorname{Re} F(\mathrm{i} R)|$ is bounded independently of $R$ while $\operatorname{Im} F(\mathrm{i} R)$ grows linearly with $R$. If one imagines drawing the graph of $\operatorname{Im} F(\mathrm{i} R)$ against $\operatorname{Re} F(\mathrm{i} R)$ (with $R$ as a parameter), the graph will start on the positive real axis and will go to infinity like the positive imaginary axis, being confined to some strip centred thereon. The origin could be on either side of this graph or the graph may encircle it, possibly many times. The total change in $\arg F(\mathrm{i} R)$, as $\mathrm{R}$ goes from zero to infinity, must be one of the values $(1-4 n) \pi / 2, n=0,1,2, \ldots$ The actual number of roots of $F(\sigma)=0$ in the right half complex plane will then be $2 n$ and $n=0$ is therefore the only possibility for stability.

The following two theorems give conditions which are sufficient (but not necessary) to have stability of the steady state $u^{*}=\ln \beta$, by ensuring that $\arg F(\mathrm{i} \infty)$ will be $\pi / 2$ in the above analysis. It will be useful to note that

$$
\begin{aligned}
& \operatorname{Re} F(\mathrm{i} R)=\tau-\tau(1-\ln \beta) \int_{0}^{\infty} f(t) \cos R t \mathrm{~d} t+d k^{2}, \\
& \operatorname{Im} F(\mathrm{i} R)=R+\tau(1-\ln \beta) \int_{0}^{\infty} f(t) \sin R t \mathrm{~d} t
\end{aligned}
$$

The first result yields stability for any convex kernel $f(t)$, the second gives stability for sufficiently small delays.

THEOREM 3.3. Let the kernel $f(t)$ satisfy $f^{\prime \prime}(t) \geqslant 0, f(\infty)=0$ and $f^{\prime}(\infty)=0$. Then the steady state $u^{*}=\ln \beta$ of (1.2) is linearly stable. 
Proof. We aim to show that $\operatorname{Re} F(\mathrm{i} R)>0$ for all $R \geqslant 0$. Integration by parts twice shows that

$$
\begin{aligned}
\int_{0}^{\infty} f(t) \cos R t \mathrm{~d} t & =-\frac{1}{R^{2}}\left(f^{\prime}(0)+\int_{0}^{\infty} f^{\prime \prime}(t) \cos R t \mathrm{~d} t\right) \\
& =\frac{1}{R^{2}} \int_{0}^{\infty} f^{\prime \prime}(t)(1-\cos R t) \mathrm{d} t \geqslant 0
\end{aligned}
$$

Now, if $\beta \leqslant \mathrm{e}^{2}$, the statement of the theorem trivially holds (by a previous theorem), so assume $\beta>\mathrm{e}^{2}$. Then $1-\ln \beta<0$ and so, since

$$
\int_{0}^{\infty} f(t) \cos R t \mathrm{~d} t \geqslant 0,
$$

it is clear that $\operatorname{Re} F(\mathrm{i} R)>0$ for all $R \geqslant 0$. In view of this fact, it is clear that the change in $\arg F(\mathrm{i} R)$, as $R$ runs from zero to infinity, can only be $\pi / 2$ and this implies that the equation $F(\sigma)=0$ will have no roots in the right half complex plane. The proof is complete.

ThEOREM 3.4. Let $\beta>\mathrm{e}^{2}$ and

$$
\tau<\frac{1}{\ln \beta-1}
$$

Then the steady state $u^{*}=\ln \beta$ of (1.2) is linearly stable.

Proof. First note that

$$
\left|\int_{0}^{\infty} f(t) \sin R t \mathrm{~d} t\right| \leqslant \int_{0}^{\infty} f(t)|\sin R t| \mathrm{d} t \leqslant R \int_{0}^{\infty} t f(t) \mathrm{d} t=R .
$$

Thus

$$
\begin{aligned}
\operatorname{Im} F(\mathrm{i} R) & \geqslant R-\tau(\ln \beta-1)\left|\int_{0}^{\infty} f(t) \sin R t \mathrm{~d} t\right| \\
& \geqslant R-\tau(\ln \beta-1) R \\
& >0 \quad \text { by hypothesis. }
\end{aligned}
$$

In view of this, it is clear that $\arg F(\mathrm{i} \infty)$ can only be $\pi / 2$ and so stability is assured. The proof is complete.

\subsection{Global stability of the non-zero steady state}

In this subsection we prove that, under the condition $\beta \in(1, \mathrm{e}]$, non-negative solutions of (1.2), posed on a finite domain with homogeneous Neumann boundary conditions, will converge uniformly to the homogeneous state $u^{*}=\ln \beta$, provided they do not start identically zero. The method is via the use of the theory of comparison principles for delay equations due to R. Redlinger. This method has also been used by Gourley and Britton [6] to study global convergence in a logistic equation with delay.

We first introduce Redlinger's [16] definition of subsolutions and supersolutions as it applies to our particular problem. A pair of suitably smooth functions $v(\boldsymbol{x}, t)$ 
and $w(\boldsymbol{x}, t)$ is called a pair of subsolutions and supersolutions for (1.2), respectively, for $(\boldsymbol{x}, t) \in \Omega \times[0, \infty)$ with the boundary condition $\nabla u \cdot \boldsymbol{n}=0$ on $\partial \Omega$ and initial conditions $u(\boldsymbol{x}, t)=\phi(\boldsymbol{x}, t)$ for $t \leqslant 0, \boldsymbol{x} \in \bar{\Omega}$, if the following conditions hold.

(i) $v(\boldsymbol{x}, t) \leqslant w(\boldsymbol{x}, t)$ for $(\boldsymbol{x}, t) \in \bar{\Omega} \times[0, \infty)$.

(ii) The differential inequalities

$$
\begin{aligned}
& \frac{\partial v}{\partial t} \leqslant-\tau v+\beta \tau\left(\int_{-\infty}^{t} f(t\right.-s) \psi(\boldsymbol{x}, s) \mathrm{d} s) \\
& \times \exp \left[-\int_{-\infty}^{t} f(t-s) \psi(\boldsymbol{x}, s) \mathrm{d} s\right]+d \nabla^{2} v \\
& \frac{\partial w}{\partial t} \geqslant-\tau w+\beta \tau\left(\int_{-\infty}^{t} f(t\right.-s) \psi(\boldsymbol{x}, s) \mathrm{d} s) \\
& \times \exp \left[-\int_{-\infty}^{t} f(t-s) \psi(\boldsymbol{x}, s) \mathrm{d} s\right]+d \nabla^{2} w
\end{aligned}
$$

hold for all functions $\psi \in C((\bar{\Omega} \times[0, \infty)) \cup(\bar{\Omega} \times(-\infty, 0]))$, with $v \leqslant \psi \leqslant w$.

(iii) $\nabla v \cdot \boldsymbol{n}=0$ and $\nabla w \cdot \boldsymbol{n}=0$ on $\partial \Omega \times[0, \infty)$.

(iv) $v(\boldsymbol{x}, t) \leqslant \phi(\boldsymbol{x}, t) \leqslant w(\boldsymbol{x}, t)$ in $\bar{\Omega} \times(-\infty, 0]$.

The following lemma can be proved by employing an argument similar to that of theorem 3.4 in Redlinger [16].

LEMMA 3.5. Let $v(\boldsymbol{x}, t)$ and $w(\boldsymbol{x}, t)$ be a pair of sub-and supersolutions for (1.2), defined as above. Suppose that $\phi \in C(\bar{\Omega} \times(-\infty, 0])$ is bounded, non-negative, uniformly Hölder continuous and $\phi_{0}(\boldsymbol{x})=\phi(\boldsymbol{x}, 0) \in C^{1}(\bar{\Omega})$. Then there exists a unique regular solution $u(\boldsymbol{x}, t)$ of the initial boundary-value problem (1.2) such that

$$
v(\boldsymbol{x}, t) \leqslant u(\boldsymbol{x}, t) \leqslant w(\boldsymbol{x}, t) \quad \text { for }(\boldsymbol{x}, t) \in \bar{\Omega} \times[0, \infty) .
$$

Next we shall prove that the solution $u(\boldsymbol{x}, t)$ is bounded above by a constant.

LEMmA 3.6. There exists a constant $K=K(\phi)>0$ such that

$$
u(\boldsymbol{x}, t) \leqslant K \quad \text { on } \bar{\Omega} \times[0, \infty) .
$$

Proof. Let $w_{0}(t)$ be the solution of the initial value problem

$$
\begin{aligned}
\frac{\mathrm{d} w_{0}}{\mathrm{~d} t} & =-\tau w_{0}+\frac{\beta \tau}{\mathrm{e}}, \quad t>0, \\
w_{0}(0) & =\sup _{s \in(-\infty, 0]} \max _{\boldsymbol{x} \in \bar{\Omega}} \phi(\boldsymbol{x}, s) .
\end{aligned}
$$

Define

$$
\bar{w}_{0}(t)= \begin{cases}w_{0}(0), & t \in(-\infty, 0] \\ w_{0}(t), & t>0\end{cases}
$$


Since $0 \leqslant \phi \leqslant \bar{w}_{0}$, it follows that $\left(0, \bar{w}_{0}\right)$ is a pair of sub- and supersolutions of (1.2) under the initial and boundary conditions. For any

$$
\psi \in C((\bar{\Omega} \times[0, \infty)) \cup(\bar{\Omega} \times(-\infty, 0]))
$$

with $0 \leqslant \psi \leqslant \bar{w}_{0}$, since $y \mathrm{e}^{-y} \leqslant 1 /$ e for $y>0$, we have

$$
\begin{array}{r}
\frac{\partial \bar{w}_{0}}{\partial t}+\tau \bar{w}_{0}-\beta \tau\left(\int_{-\infty}^{t} f(t-s) \psi(\boldsymbol{x}, s) \mathrm{d} s\right) \exp \left[-\int_{-\infty}^{t} f(t-s) \psi(\boldsymbol{x}, s) \mathrm{d} s\right]-d \nabla^{2} \bar{w}_{0} \\
\geqslant \frac{\partial \bar{w}_{0}}{\partial t}+\tau \bar{w}_{0}-\frac{\beta \tau}{\mathrm{e}}=0 .
\end{array}
$$

Thus, by lemma $3.5,0 \leqslant u(\boldsymbol{x}, t) \leqslant \bar{w}_{0}(t)$. Notice that since $\lim _{t \rightarrow \infty} \bar{w}_{0}(t)=\beta / \mathrm{e}$, it follows that there exists a constant $K=\beta / \mathrm{e}>0$ such that $\bar{w}_{0}(t) \leqslant K$ for all $t \geqslant 0$. This proves the result.

Now we can state and prove our main result on the global attractivity of the positive steady state $u \equiv \ln \beta$ of (1.2).

THEOREM 3.7. If $1<\beta \leqslant \mathrm{e}$, then any non-trivial solution $u(\boldsymbol{x}, t)$ of (1.2) with initial and boundary conditions satisfies

$$
\lim _{t \rightarrow \infty} u(\boldsymbol{x}, t)=u^{*}:=\ln \beta
$$

uniformly in $\boldsymbol{x} \in \Omega$.

Proof. Let $\bar{w}(t)$ be the solution of the initial value problem

$$
\begin{aligned}
\frac{\mathrm{d} \bar{w}}{\mathrm{~d} t} & =-\tau \bar{w}+\beta \tau \int_{-\infty}^{t} f(t-s) \bar{w}(s) \mathrm{d} s, \\
\bar{w}(\theta) & =\max _{x \in \bar{\Omega}} \phi(\boldsymbol{x}, \theta) .
\end{aligned}
$$

Then $(0, \bar{w}(t))$ is a pair of sub- and supersolutions. Thus, by lemma 3.5 , we have

$$
0 \leqslant u(\boldsymbol{x}, t) \leqslant \bar{w}(t)
$$

Since $\beta / \mathrm{e}>\ln \beta$ when $1<\beta<\mathrm{e}$ and $\beta / \mathrm{e}=\ln \beta$ when $\beta=\mathrm{e}$, we have that $\beta / \mathrm{e} \geqslant u^{*}=\ln \beta$. Now define

$$
\underline{u}(t)=\min _{\boldsymbol{x} \in \bar{\Omega}} u(\boldsymbol{x}, t), \quad \bar{u}(t)=\max _{\boldsymbol{x} \in \bar{\Omega}} u(\boldsymbol{x}, t)
$$

and let

$$
\underline{u}=\liminf _{t \rightarrow \infty} \underline{u}(t), \quad \bar{u}=\limsup _{t \rightarrow \infty} \bar{u}(t) .
$$

We shall prove that $\underline{u}=u^{*}=\bar{u}$. Theorem 2.1 and lemma 3.6 imply that

$$
0 \leqslant \underline{u} \leqslant \bar{u} \leqslant \beta / \mathrm{e} .
$$

To improve the supersolution, let $\epsilon>0$ be sufficiently small. Then, for $t_{0} \geqslant 0$, we have

$$
\bar{u}(t) \leqslant \beta / \mathrm{e}+\epsilon<1, \quad t \geqslant t_{1} .
$$


This implies that

$$
u(\boldsymbol{x}, t) \leqslant \beta / \mathrm{e}+\epsilon<1 \quad \text { on } \bar{\Omega} \times\left[t_{1}, \infty\right) .
$$

Let $w_{1}(t)$ be the solution of the initial value problem

$$
\begin{aligned}
\frac{\mathrm{d} w_{1}}{\mathrm{~d} t} & =-\tau w_{1}+\beta \tau\left(\frac{\beta}{\mathrm{e}}+\epsilon\right) \exp \left[-\left(\frac{\beta}{\mathrm{e}}+\epsilon\right)\right], \quad t>t_{1}, \\
w_{1}\left(t_{1}\right) & =\beta / e+\epsilon
\end{aligned}
$$

and define

$$
\bar{w}_{1}(t)= \begin{cases}\max \left\{\sup _{t \in\left(-\infty, t_{1}\right]} \bar{w}(t), \beta / \mathrm{e}+\epsilon\right\}, & t \in\left(-\infty, t_{1}\right], \\ w_{1}(t), & t>t_{1} .\end{cases}
$$

It follows that $\left(0, \bar{w}_{1}\right)$ is a pair of sub- and supersolutions of $(1.2)$ under the initial and boundary conditions. Denote $\nu_{1}=\beta /$ e. Since

$$
\lim _{t \rightarrow \infty} \bar{w}_{1}(t)=\beta\left(\frac{\beta}{\mathrm{e}}+\epsilon\right) \exp \left[-\left(\frac{\beta}{\mathrm{e}}+\epsilon\right)\right]
$$

and $\epsilon$ can be chosen sufficiently small, we have

$$
0 \leqslant u(\boldsymbol{x}, t) \leqslant \beta\left(\frac{\beta}{\mathrm{e}} \exp \left[-\frac{\beta}{\mathrm{e}}\right]\right)=\beta\left(\nu_{1} \exp \left[-\nu_{1}\right]\right) .
$$

Let $\nu_{2}=\beta\left(\nu_{1} \exp \left[-\nu_{1}\right]\right)$. Then $\nu_{2}<\nu_{1}$ since $\nu_{1}>u^{*}=\ln \beta$. If $1<\beta<\mathrm{e}$, then $\nu_{2}>u^{*}=\ln \beta$. Repeating the above procedure, we obtain a sequence $\left\{\nu_{n}\right\}$ satisfying $\nu_{n+1}=\beta\left(\nu_{n} \exp \left[-\nu_{n}\right]\right)$ and

$$
0 \leqslant \underline{u} \leqslant \bar{u} \leqslant \nu_{n+1}<\nu_{n}<\cdots<\nu_{1} .
$$

Thus we obtain that

$$
0 \leqslant \underline{u} \leqslant \bar{u} \leqslant u^{*}=\ln \beta
$$

since $\nu_{n} \rightarrow u^{*}$ as $n \rightarrow \infty$.

To improve the subsolution, let $\epsilon>0$ be sufficiently small so that there exists $t_{2} \geqslant 0$ such that

$$
\bar{u}(t) \leqslant u^{*}+\epsilon \quad \text { for } t \geqslant t_{2}
$$

Denote

$$
\mu_{0}=\frac{1}{2} \min \left\{\min _{t \in\left[t_{2} / 2, t_{2}\right]} \underline{u}(t), u^{*}\right\}
$$

Let $v^{\epsilon}(t)$ be the solution of the initial value problem

$$
\begin{aligned}
\frac{\mathrm{d} v^{\epsilon}}{\mathrm{d} t} & =-\tau v^{\epsilon}+\beta \tau\left(\mu_{0} \exp \left[-\mu_{0}\right]\right), \quad t>t_{2}, \\
v^{\epsilon}\left(t_{2}\right) & =\mu_{0} .
\end{aligned}
$$

Define

$$
\bar{v}^{\epsilon}(t)= \begin{cases}0, & t \in\left(-\infty, t_{2} / 2\right] \\ \left(\mu_{0} / t_{2}\right)\left(2 t-t_{2}\right), & t \in\left(t_{2} / 2, t_{2}\right] \\ v^{\epsilon}(t), & t>t_{2}\end{cases}
$$


and

$$
\bar{w}^{\epsilon}(t)= \begin{cases}\max \left\{\sup _{t \in\left(-\infty, t_{2} / 2\right]} \bar{w}(t), u^{*}+\epsilon\right\}=K, & t \in\left(-\infty, t_{2} / 2\right], \\ K+\left(\left(u^{*}+\epsilon-K\right) / t_{2}\right)\left(2 t-t_{2}\right), & t \in\left(t_{2} / 2, t_{2}\right], \\ u^{*}+\epsilon, & t>t_{2} .\end{cases}
$$

Then $\left(\bar{v}^{\epsilon}(t), \bar{w}^{\epsilon}(t)\right)$ is a pair of sub- and supersolutions. Thus

$$
\beta\left(\mu_{0} \exp \left[-\mu_{0}\right]\right) \leqslant \underline{u} \leqslant \bar{u} \leqslant u^{*}+\epsilon .
$$

Let $\mu_{1}=\beta\left(\mu_{0} \exp \left[-\mu_{0}\right]\right)$. Then $\mu_{0}<\mu_{1}<u^{*}$. Similarly, we obtain an increasing sequence $\left\{\mu_{n}\right\}$ satisfying $\mu_{n}=\beta\left(\mu_{n-1} \exp \left[-\mu_{n-1}\right]\right)$ and

$$
\mu_{0}<\mu_{1}<\cdots<\mu_{n} \leqslant \underline{u} \leqslant \bar{u} \leqslant u^{*}+\epsilon,
$$

with

$$
\lim _{n \rightarrow \infty} \mu_{n}=u^{*}
$$

This implies that

$$
\underline{u}=\bar{u}=u^{*}=\ln \beta \text {. }
$$

The case when $\beta=$ e can be treated similarly. This completes the proof.

\section{Bifurcations from the non-zero uniform state}

In this section we demonstrate that two bifurcations typically occur from the uniform steady state $u^{*}=\ln \beta$ of (1.2) as the delay $\tau$ is varied. The first of these is a bifurcation to a spatially periodic function with temporally varying amplitude, the temporal variation having been introduced via a Hopf bifurcation. At bifurcation, the spatial variation is of the form $\exp (\mathrm{i} k x)$, but the bifurcation can only happen for $k$ sufficiently small (i.e. suitably large wavelength) in a sense to be made precise.

In the literature, the two kernels $f(t)=\mathrm{e}^{-t}$ and $f(t)=t \mathrm{e}^{-t}$ are frequently considered. These are called weak and strong generic delay kernels, respectively. The use of the weak one implies that events in the past simply become exponentially less important the further one looks into the past. The strong kernel implies that a particular instant in the past is more important than any other and, with the non-dimensionalization we have in place here, this will be one time unit ago in our model.

The weak kernel $f(t)=\mathrm{e}^{-t}$ is, of course, convex. Therefore, by one of our local stability theorems, it cannot destabilize the uniform state. The strong kernel $f(t)=t \mathrm{e}^{-t}$ has Laplace transform $\bar{f}(\sigma)=1 /(1+\sigma)^{2}$, so the eigenvalue equation (3.2) determining stability of the $u^{*}=\ln \beta$ state can be written in the form

$$
\sigma^{3}+\left(\tau+2+d k^{2}\right) \sigma^{2}+\left(2 \tau+1+2 d k^{2}\right) \sigma+\tau \ln \beta+d k^{2}=0
$$

Recall that this is for perturbations of the form $\exp (\sigma t+\mathrm{i} k x)$ and we consider $k$ fixed here. To seek a Hopf bifurcation we set $\sigma=\mathrm{i} \omega$, with $\omega$ being real. This gives

$$
\omega^{2}\left(\tau+2+d k^{2}\right)=\tau \ln \beta+d k^{2} \quad \text { and } \quad \omega^{2}=2 \tau+1+d k^{2} .
$$


Combining these, we find that $\tau$ is found from the quadratic equation

$$
2 \tau^{2}+\left(5+3 d k^{2}-\ln \beta\right) \tau+d^{2} k^{4}+2 d k^{2}+2=0 .
$$

This equation has two real positive roots for $\tau$ if and only if

$$
\ln \beta>5+3 d k^{2}+\sqrt{8\left(d^{2} k^{4}+2 d k^{2}+2\right)} .
$$

Thus, if $k$ satisfies (4.3), then as $\tau$ is increased, the uniform state can lose stability through a Hopf bifurcation when $\tau$ passes through the smaller root of (4.2). The bifurcating solution will be a standing wave. However, when $\tau$ is further increased past the larger root of (4.2), the uniform state will regain stability, again through a Hopf bifurcation. To confirm that the bifurcation occurs, it is necessary to verify a transversality condition, namely, that as $\tau$ is passed through the critical value, the eigenvalues $\sigma$ cross the imaginary axis transversely. Assume that at $\tau=\tau_{0}$, $\sigma= \pm \mathrm{i} \omega_{0}$. Close to this value of $\tau$ let the eigenvalue with positive imaginary part be $\sigma(\tau)$. It satisfies

$$
\sigma(\tau)^{3}+\left(\tau+2+d k^{2}\right) \sigma(\tau)^{2}+\left(2 \tau+1+2 d k^{2}\right) \sigma(\tau)+\tau \ln \beta+d k^{2}=0 .
$$

Differentiating this implicitly with respect to $\tau$, then evaluating at $\tau=\tau_{0}$ and using $\omega_{0}^{2}=2 \tau_{0}+1+d k^{2}$ on certain terms only gives

$$
\sigma^{\prime}\left(\tau_{0}\right)=\frac{-\ln \beta-2 \mathrm{i} \omega_{0}+\omega_{0}^{2}}{-2 \omega_{0}^{2}+2 \mathrm{i} \omega_{0}\left(\tau_{0}+2+d k^{2}\right)} .
$$

Taking the real part,

$$
\operatorname{Re} \sigma^{\prime}\left(\tau_{0}\right)=\frac{\ln \beta-5-4 \tau_{0}-3 d k^{2}}{2\left(\omega_{0}^{2}+\left(\tau_{0}+2+d k^{2}\right)^{2}\right)} .
$$

Now $\tau_{0}$ is either of the two roots of the quadratic

$$
q(\tau):=2 \tau^{2}+\left(5+3 d k^{2}-\ln \beta\right) \tau+d^{2} k^{4}+2 d k^{2}+2=0 .
$$

We claim that $\operatorname{Re} \sigma^{\prime}\left(\tau_{0}\right)>0$ when $\tau_{0}$ is the smaller of these roots, and $\operatorname{Re} \sigma^{\prime}\left(\tau_{0}\right)<0$ when $\tau_{0}$ is the larger. To show this, it is enough to formally set $\operatorname{Re} \sigma^{\prime}\left(\tau_{0}\right)=0$ and show that the value of $\tau_{0}$ at which this happens is in between these two roots. A simple graphical argument will show that it is enough to show that $q\left(\left(\ln \beta-5-3 d k^{2}\right) / 4\right)<0$. After some algebra, this is equivalent to showing that $\left(\ln \beta-5-3 d k^{2}\right)^{2}>8\left(d^{2} k^{4}+2 d k^{2}+2\right)$ and we are, in fact, already assuming this in $(4.3)$.

This confirms that Hopf bifurcations occur but does not establish whether the bifurcations are sub or supercritical.

Another bifurcation that can occur in (1.2) from its uniform state $u^{*}=\ln \beta$ is a bifurcation to periodic travelling wave solutions. All we shall do here is determine the bifurcation curve in the $(\tau, c)$ parameter plane along which this occurs, where $c$ is the speed of the wave. If we convert (1.2) into travelling wave form by writing $u(x, t)=U(z)=U(x-c t)$, and then linearize by setting $U(z)=\ln \beta+V(z)$, the result is

$$
d V^{\prime \prime}+c V^{\prime}-\tau V+\tau(1-\ln \beta) \int_{0}^{\infty} f(\xi) V(z+c \xi) \mathrm{d} \xi=0
$$




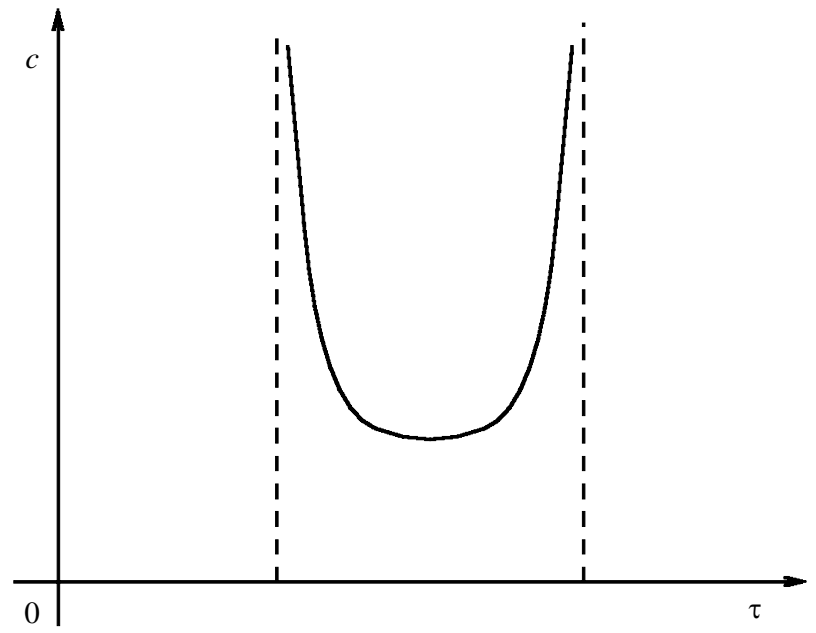

Figure 1. Bifurcation curve in the $(\tau, c)$ parameter plane, for the bifurcation to periodic travelling wave solutions from the uniform state $u^{*}=\ln \beta$ of (1.2).

To seek a periodic travelling wave, set $V=\exp (\mathrm{i} \omega z)$. This gives

$$
-d \omega^{2}+c \omega \mathrm{i}-\tau+\tau(1-\ln \beta) \bar{f}(-c \omega \mathrm{i})=0,
$$

$\bar{f}$ being the Laplace transform of $f$. The bifurcation can only occur for the strong generic kernel $f(t)=t \mathrm{e}^{-t}$. For this case, equating real and imaginary parts in (4.4) will give $\tau$ and $c$ in terms of $\omega$. The result is, provided $\ln \beta>9$,

$$
\begin{aligned}
\tau & =\frac{1}{4}\left[\ln \beta-5 \pm \sqrt{(\ln \beta)^{2}-(\ln \beta)\left(10+8 d \omega^{2}\right)+9+8 d \omega^{2}}\right]-d \omega^{2}
\end{aligned}
$$

for $0<\omega^{2} \leqslant(\ln \beta-9) / 8 d$. These equations define a curve in the $(\tau, c)$ parameter plane (shown in figure 1) and the bifurcation will occur as this curve is crossed.

\section{Discussion}

We have studied a version of Nicholson's blowflies equation that is more general than the versions studied by Yang and So $[19,22]$ by incorporating the maturation delays by way of an integral convolution over all past times (a distributed delay) which we have studied as far as possible for general delay kernels $f(t)$. Using mainly the principle of the argument applied to the linearized equation, we have obtained various conditions which are sufficient to ensure the linearized stability of the nonzero uniform steady state. One consequence is that no convex kernel can destabilize this uniform state.

We have also studied the global convergence of solutions and have proved global convergence to the zero state under the condition $\beta<1$, a condition that cannot be improved since the zero state is linearly unstable if the inequality is reversed.

By employing a theory of sub- and supersolutions for delay differential equations, we were able to show, by successively improving pairs of sub- and supersolutions for 
our equation, that global stability of the non-zero uniform state is assured under the condition $1<\beta \leqslant \mathrm{e}$. The weaker condition $1<\beta \leqslant \mathrm{e}^{2}$ guarantees linear stability of the same state.

We also studied the case when the non-dimensionalized delay kernel assumes the special form $t \mathrm{e}^{-t}$, the strong generic case, and noted that two bifurcations are possible, both of which are associated with temporal oscillations in the kinetics. These bifurcations are to periodic standing wave solutions and periodic travelling waves (wavetrains) and which ones will be observed will in practice depend on the boundary conditions (if any). If bifurcation occurs at all, there are two bifurcation points; the uniform $\ln \beta$ state is stable both for sufficiently small and sufficiently large delays. It will be interesting to investigate the stability of, in particular, the periodic travelling wave solutions since it was shown by Gourley and Britton [7], for a modified logistic equation with time delays (and non-local spatial effects too), that small-amplitude periodic travelling waves arising via bifurcation from a uniform state are unstable. Results of this kind are also known for the special class of reaction-diffusion systems known as $\lambda-\omega$ systems (see [10]). We would conjecture that our wavetrains are probably only stable when their amplitude exceeds a certain critical value. Further investigation of the stability of these solutions is presently in progress.

\section{Acknowledgments}

The research of S.R. was supported in part by the Natural Science and Engineering Research Council of Canada.

\section{References}

1 L. L. Bonilla and A. Liñán. Relaxation oscillations, pulses, and traveling waves in the diffusive Volterra delay-differential equation. SIAM J. Appl. Math. 44 (1984), 369-391.

2 N. F. Britton. Reaction-diffusion equations and their applications to biology (Academic, 1986).

3 N. F. Britton. Spatial structures and periodic traveling waves in an integro-differential reaction-diffusion population model. SIAM J. Appl. Math. 50 (1990), 1663-1688.

4 J. M. Cushing. An operator equation and bounded solutions of integrodifferential systems. SIAM. J. Math. Analysis 6 (1975), 433-445.

5 J. M. Cushing. Integrodifferential equations and delay models in population dynamics (Springer, 1977).

6 S. A. Gourley and N. F. Britton. On a modified Volterra population equation with diffusion. Nonlinear Analysis 21 (1993), 389-395.

7 S. A. Gourley and N. F. Britton. Instability of travelling wave solutions of a population model with nonlocal effects. IMA J. Appl. Math. 51 (1993), 299-310.

8 W. S. C. Gurney, S. P. Blythe and R. M. Nisbet. Nicholson's blowflies revisited. Nature 287 (1980), 17-21.

9 G. E. Hutchinson. Circular cause systems in ecology. Ann. NY Acad. Sci. 50 (1948), 221246.

10 N. Kopell and L. N. Howard. Plane wave solutions to reaction-diffusion equations. Stud. Appl. Math. 52 (1973), 291-328.

11 M. R. S. Kulenović, G. Ladas and Y. G. Sficas. Global attractivity in Nicholson's blowflies. Appl. Analysis 43 (1992), 109-124.

12 R. K. Miller. Asymptotic stability and perturbations for linear Volterra integrodifferential systems. In Delay and functional differential equations and their applications (ed. K. Schmitt), pp. 257-268 (Academic, 1972). 
13 J. D. Murray. Mathematical biology (Springer, 1989).

14 A. J. Nicholson. An outline of the dynamics of animal populations. Austral. J. Zoo. 2 (1954), 9-65.

15 R. M. Nisbet and W. S. C. Gurney. Modelling fluctuating populations (Wiley, 1982).

16 R. Redlinger. On Volterra's population equation with diffusion. SIAM J. Math. Analysis 16 (1985), 135-142.

17 H. L. Smith. Monotone dynamical systems, an introduction to the theory of competitive and cooperative systems (Providence, RI: American Mathematical Society, 1995).

18 J. W.-H. So and J. Yu. Global attractivity and uniform persistence in Nicholson's blowflies. Diff. Eqns Dynam. Syst. 2 (1994), 11-18.

19 J. W.-H. So and Y. Yang. Dirichlet problem for the diffusive Nicholson's blowflies equation. J. Diff. Eqns 150 (1998), 317-348.

20 J. W.-H. So, J. Wu and Y. Yang. Numerical steady-state and Hopf bifurcation analysis on the diffusive Nicholson's blowflies equation. Appl. Math. Comput. 111 (2000), 33-51.

$21 \mathrm{~J}$. Wu. Theory and applications of partial functional differential equations (Springer, 1996).

22 Y. Yang and J. W.-H. So. Dynamics for the diffusive Nicholson's blowflies equation. In Dynamical systems and differential equations (ed. W. Chen and S. Hu), vol. II, pp. 333352 (Springfield, MO: Southwest Missouri State University, 1998).

(Issued 15 December 2000) 